\title{
Multi-loop Model Reference Adaptive PID Control for Fault-Tolerance
}

\author{
B. B. ALAGOZ, G. KAVURAN, A. ATES, C. YEROGLU and H. ALISOY
}

\begin{abstract}
This study demonstrates an application of multiloop Model Reference Adaptive Control (MRAC) structure for enhancement of fault tolerance performance of closed-loop PID control systems. The presented multi-loop MRAC-PID control structure can be used to transform a conventional PID control system to an adaptive control system by combining an outer adaptation loop. This study shows that the proposed control structure can improve fault tolerance and fault detection performance of the existing closed-loop PID control systems without modifying any coefficients of PID controllers, and this asset is very useful for increasing robust control performance of the existing industrial control systems. This advantage originates from the reference input shaping technique that is implemented to combine adaptation and control loops. Numerical and experimental studies are presented to illustrate an application of the MRAC-PID control structure for rotor control applications.
\end{abstract}

Index Terms - Adaptive Control, Fault detection, Fault tolerant control, Fault diagnosis.

\section{INTRODUCTION}

Q Yontrol systems are mainly designed according to mathematical models of controlled systems. The dependence on mathematical modeling is one of the factors, which leads to degradation of long-term control performance of static controller structures in real world control applications.

BARIS BAYKANT ALAGOZ, is with Department of Computer Engineering, Inonu University, Malatya, Turkey,(e-mail: baykant.alagoz@inonu.edu.tr).

(iD) https://orcid.org/ 0000-0001-5238-6433

GURKAN KAVURAN, is with Department of Mechatronics Engineering, Firat University, Elazig, Turkey, (e-mail: gurkankavuran@gmail.com).

iD https://orcid.org/ 0000-0003-2651-5005

ABDULLAH ATES, is with Department of Computer Engineering, Inonu University, Malatya, Turkey (e-mail: abdullah.ates@ inonu.edu.tr).

(iD https://orcid.org/ 0000-0002-4236-6794

CELALEDDIN YEROGLU, is with Department of Computer Engineering, Inonu University, Malatya, Turkey (e-mail: c.yeroglu @ inonu.edu.tr).

(iD https://orcid.org/ 0000-0002-6106-2374

HAFIZ ALISOY, is with Department of Electronics and Communication Engineering, Namik Kemal University, Tekirdag, Turkey, (e-mail: halisoy@nku.edu.tr).

iD https://orcid.org/ 0000-0002-9695-5594

Manuscript received April 24, 2019; accepted July 6, 2019.

DOI: $10.17694 /$ bajece. 557674
Unpredictable temporal alterations can change in system behavior in real-world applications, and they generally cause change of system models. Model uncertainties and limitations of mathematical modeling methods decrease long-term validity of system models. In fact, component and material ageing or structural faults are inevitable and they leads to change of system models. Consequently, detection of performance deteriorations and corresponding retuning efforts of controller parameters are required to maintain long-term control performance under real world conditions. Instead of static controller structures, which cannot respond to those alterations, the flexible systems, which can adapt themselves for changing conditions and dynamics of controlled systems, can be more effective to achieve long-term real control performance. This type of flexible control systems are widely referred to as adaptive control systems so that they are capable of adapting themselves for changing operating conditions.

In addition to the external factors, namely environmental disturbances, there are also structural factors that can lead to fluctuation of model parameters depending on a number of temporal phenomenon, such as ageing of materials, failure of system components etc. Specifically, process engineers observed that automated systems can be vulnerable to component faults, defects in sensors or actuators in the process or even in the controllers. Some of these faults cannot be tolerated by closed-loop control systems and they can develop into the malfunction of the control loop [1]. This can be a serious problem, particularly for remote or mission-critical control applications, e.g. remote or interplanetary missions. In these control applications, spontaneous controller tuning efforts may risk the remote missions. The present study illustrates an application of the adaptive control scheme, which may reduce need for retuning efforts in feedback controllers.

Unmanned air vehicles (UAV) with electrical multi-rotor platforms can present several advantages for remote and planetary search missions inside gaseous or liquid environments. In these environments, unknown and complicated ground obstacles can be a problem for effective operation of unmanned ground vehicles. Multi-rotor UAVs can easily fly over blocking ground obstacles and trip over surface more energy-efficiently when compared to operation of unmanned ground vehicles. Performance of multi-rotor UAVs strongly depends on rotor control performance, and fault tolerance and adaptation skill are key assets for success of these type remote applications. 
Due to their proven performance in real applications, PID controllers have become an industrial standard, and they are frequently used in UAV control applications [2-3]. PID controllers are simple in implementation and yet effective to respond control requirements of practical applications [4]. However, the constant coefficient PID controllers are quite vulnerable to system perturbations that may originate from external or structural factors. When a system perturbation occurs, they need retuning of controller coefficients to maintain control performance. Since increasing risks of instability and crash of UAVs while performing real-time retuning or online self-tuning actions, update of PID coefficients in operation may not be viable and secure for mission-critical applications. In this sense, motivation of the present study is to improve robust performance of PID control systems without need for retuning actions and this is performed by collaboration MIT rule of MRAC loop. Input shaping technique is used to combine existing PID control loop and MRAC loop because it does not need retuning of PID controller coefficients. The adaptation is performed by shaping the reference input by MRAC loop.

Researches on adaptive control system topic can be traced back to mid-sixties [5] and adaptive control structures have been studied for improvement of robust performance of flight control systems. Discussions on MRAC approaches initiated in late 70s [6]. Later, it has become a major topic of control literature [7-12]. MRAC approaches have been reported to improve robust performance of control systems [12] and therefore they have been widely utilized in the case that system models were inexact or prone to perturbations.

MRAC structures are essentially taken into account as an active control system, of which gain parameters are adjusted by update rules when system dynamics are altered [13-14]. A flight control system should deal with complicated aircraft dynamics, system nonlinearities and frequent variability of flight conditions depending on altitude, payload and weather conditions. One of the solutions is to use reference model based adaptive control strategies that aim to approximate response of reference model to maintain flight control performance under varying conditions of flights [15]. In this manner, we prefer MRAC-PID structures for adaptive rotor control application and investigate possible contributions of this structure to robust control performance. Recently, MRAC has been utilized in many complicated control problems such as for adaptive system control based on only input-output measurements [16], for uncertain switched systems with unmodeled input dynamics [17]. By comparing with performance of PID control, performance improvement of MRAC has been shown in extremum seeking control of photovoltaic systems [18].

A well-known and basic MRAC implementation uses gradient descent optimization for control law derivation, which was commonly referred to as MIT rule $[9,11]$. This control rule was developed at Massachusetts Institute of Technology (MIT) in 1960 [11]. Essentially, the solution of MIT rule continuously follows descending of gradient trends of model approximation errors while updating gain parameters of control systems. Although it cannot control stable plants, computational complexity of the method is not high and its cooperation with other controller structure can improve adaptation skill of conventional control systems [19-22].

Fault-tolerant control is an important topic that has been widely discussed for industrial control applications [23] and flight control systems [24-25]. Blanke et al. have been defined the fault tolerance of control systems as "the ability of a controlled system to maintain its control objectives despite the occurrence of a fault" [1]. Particularly, main goal of faulttolerant control is to prevent local faults to develop into system malfunctions that fail safety-critical or mission-critical control applications. For these applications, degradation in control performance cannot be tolerable, and a certain degree fault-tolerance is achievable by reconfiguring controller parameters for new conditions [1]. In literature, mainly, supervised automation schemes have been proposed to diagnose and recover fault status in control systems. These schemes may need additional blocks to manage detection and diagnosis process and these blocks take action for reconfiguring or retuning of the dynamic systems [26-27]. Retuning effort, while performing control actions, is not safe because it may cause short-time instability and control performance degradations.

In our previous work, multi-loop MRAC fractional-order PID control structure (MRAC-FOPID) has been theoretically investigated and improvement of fault tolerance skills of fractional-order PID control systems has been shown numerically [28]. Then, the multi-loop MRAC-FOPID control structure has been applied for control of experimental magnetic levitation system in order to demonstrate improvement in disturbance rejection performance of magnetic levitation control system [29]. Afterward, this method has been extended to additional loops and control performance improvements of this multi-loop approach has been shown for speed control of a servo plant [30]. The current study demonstrates performance of multi-loop MRAC-PID control structure to enhance fault tolerance and fault diagnosis capabilities of experimental electrical rotor PID control system. Since PID control is the most widely utilized industrial controller structure, demonstration of improvement in performance of PID control system can be very beneficial for industrial control applications.

\section{Methodology}

\section{A. Preliminary Knowledge for Application of MRAC-PID Structure}

Our control objective is to improve fault tolerance control performance of existing closed loop PID control systems by appending a MIT rule. For this purpose, the following twostep design methodology [28-29] was applied for MRAC-PID rotor control system:

Step 1: A transfer function model for an existing and operating closed loop control system is obtained by applying closed loop model identification, and then the transfer function of the closed loop control system is used as a reference model of MRAC loop.

Step 2: The closed loop PID control system is combined with the MRAC loop according to input shaping technique. 
These tasks form a hierarchically connected two-feedback loops such that the one is inner loop, which is the existing closed loop PID control system and performs control actions and stability, and the other is outer loop, which only performs MIT rule to perform adaptation according to reference model. Accordingly, while the inner loop with PID control works for system stability, the outer loop with MIT rule works for approximation of response of the closed loop control system to the reference model, which is namely adaptation. Thus, a change in control performance of inner loop can be easily detected by observing model error signal and then this mismatch can be tolerated by contributions of the MIT rule.

Multi-loop control architectures have been addressed previously to improve certain weaknesses of conventional PID systems, and related works indicates potentials of multi-loop control structures to improve control performance [28-32]. Majority of these studies aim retuning of PID controller coefficients according to the model mismatch error. The proposed multi-loop MRAC-PID system does not tune any coefficients of the existing PID controller. Instead, an additional MIT rule as an outer loop is connected to reference input of the closed loop PID control systems. This additional loop only performs for shaping reference input of inner loop so that the response of the inner loop approximates to the response of the reference model. Thus, it can restore control performance of the closed loop PID control system (inner loop) according to response of the reference model.

In the current study, the reference model is taken as the transfer function model of well-tuned and operating closed loop PID rotor control system. This reference model represents a fault-free, normal state of the PID control system and it is used as a memory of normal system status. Thus it keeps the system input-output knowledge of the fault-free state (normal state) of the system and this knowledge is used for restoration of control performance in case of a fault state. This approach is very useful for maintaining initial and faultfree control performance of the existing PID control systems. It does not need any retuning efforts of PID controllers.

In the current study, it is assumed that fault events are not determined, however faults cause a change in the PID control system responses. Sometimes, a closed-loop control system can hide minor faults and these faults may not be detected until they develop a control-loop failure [1]. To deal with this problem, model mismatch error of the proposed MRAC-PID structure is useful for fault detection and monitoring: A change in closed loop control system (inner loop), which leads to a change in response of inner loop, is detectable by observing model error signal because the model error signal shows divergences of the closed loop control system response from its normal, fault-free responses that were retrieved by the reference model. Then, MIT rule takes action to decrease the magnitude of model error signal by applying reference inputshaping to closed loop control system. This enforces response of control system to approximate response of the reference model. Thus, it can recover control performance at certain degrees. This is the underlying mechanism of MRAC-PID structure that increases fault-tolerance and detection skills of conventional PID control system.

\section{B. Theoretical Background and Fault Modeling}

Figure 1 shows a block diagram of the proposed multi-loop MRAC-PID system. The inner loop performs closed loop PID control according to the control error $e_{c}=u_{r}-y$, and outer loop, which employs the MIT rule for adaptation of the system by using the model error $e_{m}=y-y_{m}$. Let us denote the transfer function of PID controller, $C(s)=k_{p}+\frac{k_{i}}{s}+k_{d} s$, and the controlled plant, $G(s)$. The transfer function of the inner loop can be expressed in the form of

$$
T(s)=\frac{C(s) G(s)}{1+C(s) G(s)}
$$

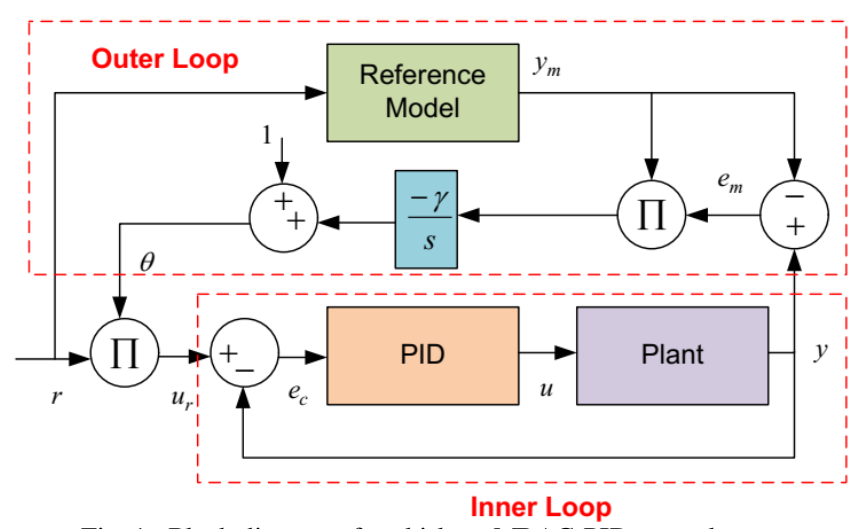

Fig. 1. Block diagram of multi-loop MRAC-PID control system

To maintain initial performance of the closed loop PID control system, the reference model is taken as the transfer function of inner loop when the PID control system is in normal, well tuned and fault-free state. Accordingly, transfer function of reference model can be expressed as,

$$
T_{m}(s)=\frac{C_{o}(S) G_{o}(S)}{1+C_{o}(S) G_{o}(S)}
$$

where $C_{o}(s)$ and $G_{o}(s)$ are transfer functions of the controller and plant in normal operation state. Adaptation of system is carried out by shaping reference input of the inner loop according to $u_{r}=\theta r$. The adaptation gain $\theta$ is determined according to MIT rule, which is based on gradient descent optimization of the cost function $J$ given by,

$$
J=\frac{1}{2} e_{m}^{2}
$$

The MIT rule for MRAC [7-9] was commonly expressed in the form of

$$
\frac{d \theta}{d t}=-\gamma \frac{d J}{d \theta}
$$


By taking Laplace transform of equation (4), it is written as follows,

$$
\theta=-\gamma \frac{1}{s} \frac{d J}{d \theta}=-\gamma \frac{1}{s} e_{m} \frac{d e_{m}}{d \theta}
$$

One can consider the model error and reference input in the form

$$
e_{m}=y-y_{m}=T(s) u_{r}-T_{m}(s) r=T(s) \theta r-T_{m}(s) r
$$

and $r=y_{m} / T_{m}(s)$, respectively. Then, the sensitivity derivate of the system is written as,

$$
\frac{d e_{m}}{d \theta}=\frac{T(s)}{T_{m}(s)} y_{m}
$$

By using equation (6) in equation (5), MIT rule for the update of adaptation gain $\theta$ to minimize cost function $J$ is obtained as,

$$
\theta=-\gamma \frac{1}{s}\left(\frac{T(s)}{T_{m}(s)} y_{m} e_{m}\right) .
$$

A bias value of 1 is added to allow a faster build-up of the adaptation gain $\theta$ when the system is initiated. Otherwise, delay of reference model leads to a zero value of $\theta$ for while and it prolongs transient regime of the control system at the system start-up.

$$
\theta=-\gamma \frac{1}{s}\left(\frac{T(s)}{T_{m}(s)} y_{m} e_{m}\right)+1
$$

Let us investigate the normal and faulty cases for this system [28]:

* In case of normal operation, $T(s)=T_{m}(s)$, the adaptation gain $\theta$ becomes,

$$
\theta=-\gamma \frac{1}{s} y_{m} e_{m}+1
$$

* In case of fault or parametric perturbation, $T(s) \neq T_{m}(s)$, and one can consider two circumstances;

(i) The fault comes from plant function, in this case, one can consider the cases of $G(s) \neq G_{o}(s)$ and $C(s)=C_{o}(s)$, the adaptation gain $\theta$ goes to the value of,

$$
\theta=-\gamma \frac{1}{s}\left(\frac{\Delta_{o}(s) G(s)}{\Delta(s) G_{o}(s)}\right) y_{m} e_{m}+1
$$

(ii) The fault comes from PID controller, in this case $G(s)=G_{o}(s)$ and $C(s) \neq C_{o}(s)$, the adaptation gain $\theta$ goes to the value of,

$$
\theta=-\gamma \frac{1}{s}\left(\frac{\Delta_{o}(s) C(s)}{\Delta(s) C_{o}(s)}\right) y_{m} e_{m}+1
$$

where the $\Delta(s)=1+C(s) G(s)$ and $\Delta_{o}(s)=1+C_{o}(s) G_{o}(s)$ are characteristic polynomials of transfer functions of closed loop PID control system in faulty case and in normal case, respectively. The fault diagnosis can be carried out by observing value of $\theta$ signal. In case of normal operation $\left(T(s)=T_{m}(s)\right)$, the condition $e_{m}=0$ should be satisfied:

$$
e_{m}=T(s) \theta r-T_{m}(s) r=T_{m}(s)(\theta-1) r=0
$$

This equation can be always valid in case of $\theta-1=0$. Here, the reference signal $r$ changes independent of the system. So, the normal operation of system (fault-free status) can be detected by the state of $\theta=1$. Accordingly, the faulty case of system can be detected by the case of $\theta \neq 1$ because it yields a non-zero model error. Two types of fault models are investigated [28]:

i) Gain faults: The gain fault case can be modeled as $T(s)=k T_{m}(s)$, where the state of $k \neq 1$ is referred to as the gain fault of the system. The case of $k=1$ infers normal state of system. By rearranging the equation (12) for this type of fault, one solves $T_{m}(s)(k \theta-1) r=0$ and obtains the $\theta=1 / k$ for recovery the system, which provides $e_{m}=0$. Hence, any gain type fault case is detected by observing $\theta$ signal for a value of $k=1 / \theta$.

ii) Structural factorable faults: This case can be modeled as $T(s)=F(s) T_{m}(s)$, where the $F(s)$ is called transfer function of structural factored type fault. When equation (12) is rearranged for this fault case as $T_{m}(s)(F(s) \theta-1) r=0$, one observes evolution of the adaptation gain, which recovers fault case, as $\theta=F(s)^{-1}$. For instance, let's assume that a structural fault causes an additional system pole with a time constant $\tau_{f}$, the fault model becomes $F(s)=1 /\left(\tau_{f} s+1\right)$. In this circumstance, when this fault case is restored by MIT rule, structural faults can be detected by a $\theta$ response that is expressed by $\theta=F(s)^{-1}=\left(\tau_{f} s+1\right)$.

\section{SimUlation STUDY}

This section presents a numerical study to demonstrate application of multi-loop MRAC-PID control for rotor control. Simulation results were obtained by using numerical model of experimental rotor control test platform in 
MATLAB/Simulink environment. The experimental system was tuned and controlled by a closed loop PID control system. In well-tuned and fault-free state, the closed loop model identification was carried out by means of model identification toolbox of MATLAB. The following transfer function of closed loop PID control system was identified,

$$
\begin{gathered}
-59.91 s^{8}+526.2 s^{7}+1257 s^{6}+9211 s^{5}+6795 s^{4} \\
+2748 s^{3}+569.8 s^{2}+68.68 s+5.187 \\
s^{10}+134.3 s^{9}+934.7 s^{8}+3890 s^{7}+1.01 .10^{4} s^{6}+1.395 .10^{4} s^{5}+7846 s^{4} \\
+2732 s^{3}+566 s^{2}+66.44 s+5.195
\end{gathered}
$$

After modeling of tuned closed loop control system in faultfree state, the transfer function of the model, given by equation (13), was implemented as the reference model of the MRAC-PID control system. Then, the MRAC-PID system, which is depicted in Fig. 8, was implemented by appending outer loop (MRAC with MIT rule) to inner control loop (closed loop PID control system). The adaptation rate $\gamma$ was set to 1 in simulation and experimental studies to provide enough fast system response in our tests. It should be noted that too large values of $\gamma$ can lead to the instability of system. PID controller coefficients were not changed during simulation and experiments. Simulation study was performed for $10000 \mathrm{sec}$ by applying square wave reference signal so that we can observe long-term system responses for low and high frequency components of square wave reference signal. Figures 2 compares simulation results, which are obtained for the proposed multi-loop MRAC-PID structure and the conventional PID control system in case of a gain fault of the closed loop PID control system, which is expressed as $T(s)=0.7 T_{m}(s)$. This synthetic fault state is initiated at 5000 sec by instantly switching DC gain of $T(s)$ function from 1 to 0.7. At the beginning of the fault, divergence of the multi-loop MRAC-PID system from reference model is shown in Fig. 3.

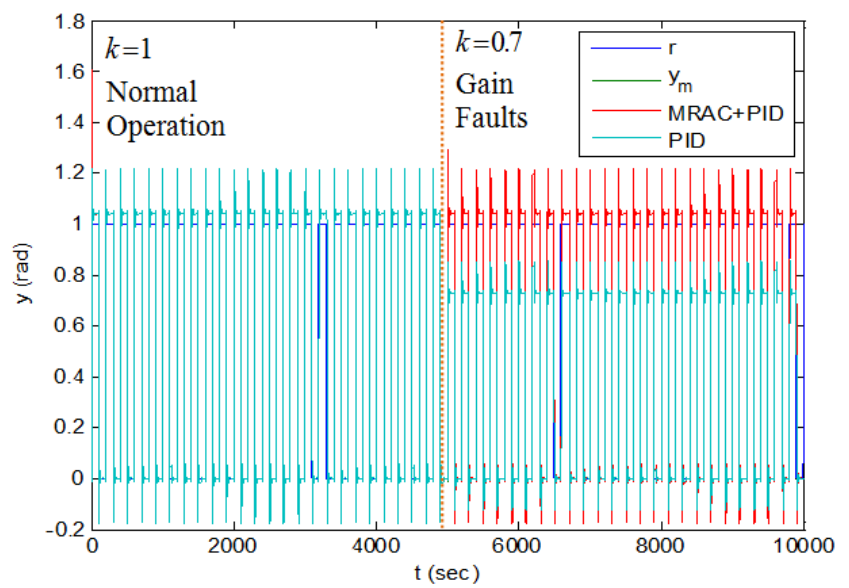

Fig. 2. Time responses of multi-loop MRAC-PID system and conventional PID control system for the case of gain fault of the closed loop PID control system at $5000 \mathrm{sec}$.

Fig 3 shows a close view of simulation results around 5000 sec in Fig 2. However, the response of multi-loop MRAC-PID system can be restored in $10 \mathrm{sec}$, and therefore the output of multi-loop MRAC-PID system ( $y)$ is the same as the output of reference model $\left(y_{m}\right)$ in Fig. 4. Whereas, the conventional PID control yields a steady state error. These results clearly show that the proposed system can deal with the gain fault of $k=0.7$.

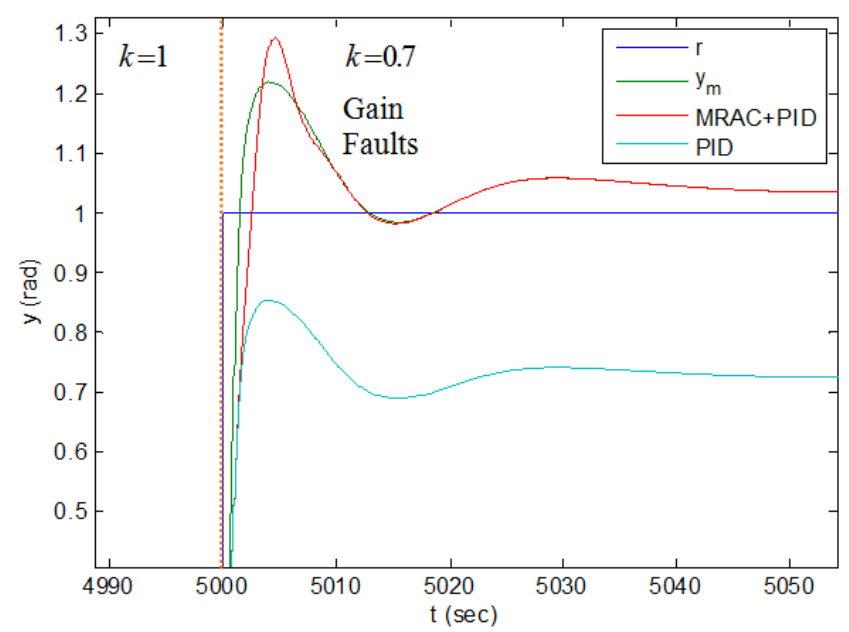

Fig. 3. A close view of Fig.2 to show responses of both control systems at the beginning of gain fault state at $5000 \mathrm{sec}$.

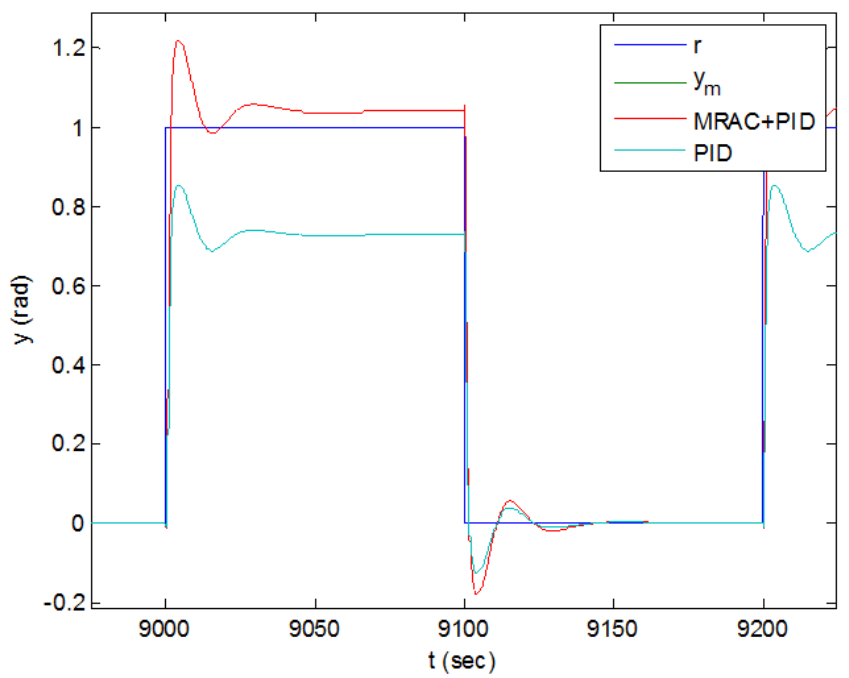

Fig. 4. A close view of Fig.2 to show responses of both control systems at $9000 \mathrm{sec}$.

In Fig. 5, it was observed that the adaptation parameter $\theta$ settles to the value of 1.4 , which is very consistent with the result of theoretical formula, $\theta=1 / k=1.4$, for gain type fault of closed loop transfer function. As expected, in normal (faultfree) case before the fault insertion at simulation time of 5000 sec, parameter $\theta$ settles to the value of 1 to indicate normal status of the system. Hence, the level of $\theta$ signal can be utilized to monitor gain type faults: One can define a gain fault index $k=1 / \theta$ to detect gain type fault case. In this simulation, this index was accurately detected as $k=1 / 1.4=0.7$ and $T(s)=0.7 T_{m}(s)$ fault case was diagnosed by monitoring level of $\theta$. Fig. 6 shows the evolution of model 
error $e_{m}$ during adaptation process. The figure clearly demonstrates the adaptation of the proposed system by settling of model error $e_{m}$ to 0 again in $10 \mathrm{sec}$.

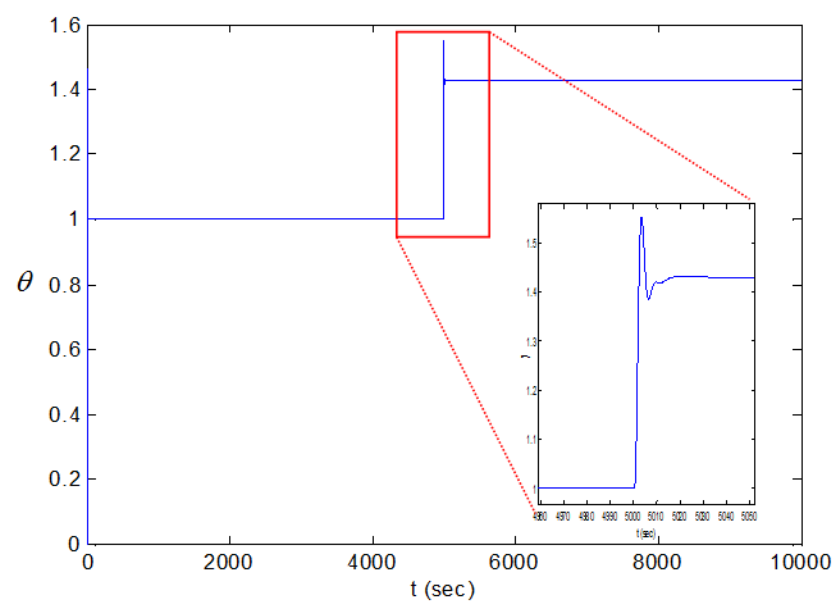

Fig.5. Change of $\theta$ at gain fault state.

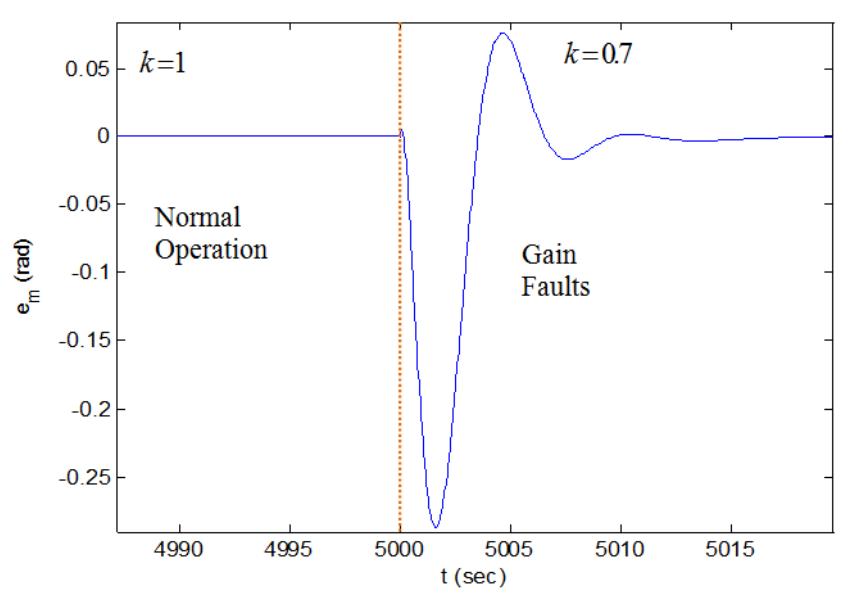

Fig.6. Change of $e_{m}$ at the beginning of gain fault state.

\section{EXPERIMENTAL STUDY}

Figure 7 shows a picture of rotor control test platform that was built for adaptive rotor control research studies. The system is composed of an electrical rotor, a rotary encoder, a shaft and a control card. The rotor hovers and the angle of shaft is controlled by the feedback of rotary encoder. Matlab/simulink environment was used to perform all tasks: control system simulation and design, digital implementation of the design on control card, monitoring of this experimental platform. Figure 8 shows an implementation of multi-loop MRAC-PID system and fault insertion model in experimental study. For normal (fault-free) state of the system, the fault model $F(s)$ was set to 1 . Figures 9 and 10 demonstrate responses of the proposed multi-loop MRAC-PID system and the conventional PID control system for a type gain fault insertion, $T(s)=0.7 T_{m}(s)$ , for experimental closed loop PID control system. This synthetic fault state was generated at $120 \mathrm{sec}$ by switching
$F(s)=1$ to $F(s)=0.7$. The figures clearly show that the output of multi-loop MRAC-PID system can approximate to the output of reference model in about $30 \mathrm{sec}$ period, and the system can deal with the DC gain fault $k=0.7$. But, conventional PID control system cannot deal with this fault type case of the PID control system. For the gain fault of $k=0.7$, theoretical formula suggests $\theta=1 / k=1.4$ for adaptation action.

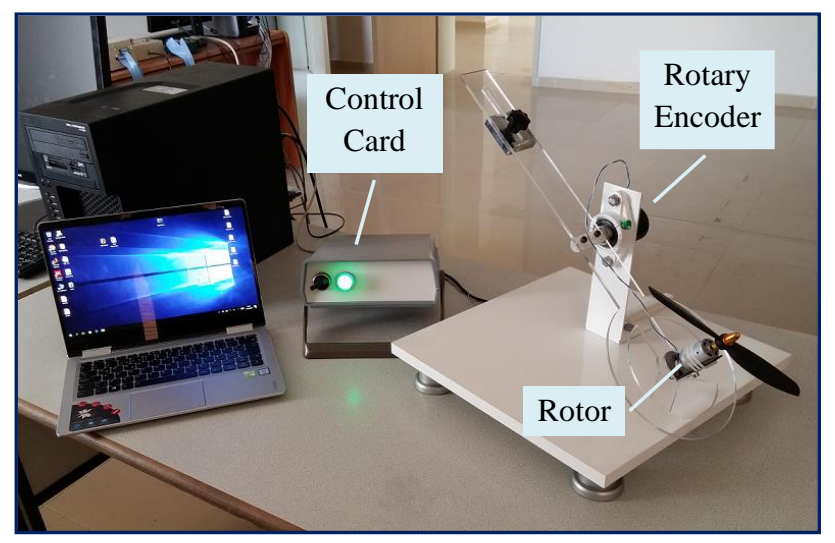

Fig. 7. A picture of experimental rotor control test platform

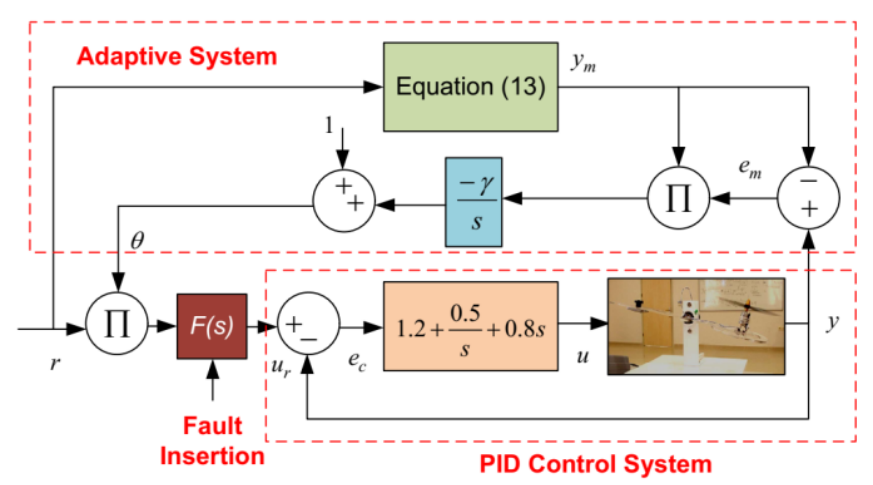

Fig. 8. Block diagram of multi-loop MRAC-PID system and fault insertion model for experimental study.

Figure 11 confirms this result by showing increase of $\theta$ up to the level of 1.4. In fault-free normal operation, the parameter $\theta$ stays at around the value of 1 in Fig. 11. These experimental results confirm that level of $\theta$ can be used to diagnose DC gain fault states of closed loop control systems. Fig. 12 shows the evolution of $u_{r}$ during the adaptation process. The $u_{r}$ signal was formed by shaping of the reference input $r$ by MIT rule and it adjusts level to $r$ to tolerate impacts of gain fault. Fig. 13 presents the evolution of model error that convergences again to zero after the recovery of the fault case. These results clearly show that gain type fault case can be easily diagnosed by monitoring $\theta$ signal and the proposed system can restore again the system response based on the response of the reference model. Experimental results are also in well-agreement with simulation results and theoretical analyses. Figures 14, 15 and 16 show test results of 
the proposed multi-loop MRAC-PID system and conventional PID control system. We assumed that this structural fault results in an additional real system pole with a time constant $\tau_{f}=3 \mathrm{sec}$ and it can be expressed as $F(s)=1 /(3 s+1)$. This synthetic fault case was initiated at $120 \mathrm{sec}$ by connecting one pole $F(s)$ function to system. Figure 15 and 16 show that the multi-loop MRAC-PID system adapts itself for this fault case and approximates to the response of the reference model. Figure 16 shows the change of $\theta$ and Figure 17 shows the evolution of $u_{r}$ during the adaptation process. Both experimental results show that multi-loop MRAC-PID system can approximate to response of the reference model in cases of these fault models and verify adaptation skill of the proposed MRAC-PID structure. Conventional PID control system more diverged from initial well-tuned system response (reference model response) in fault cases.

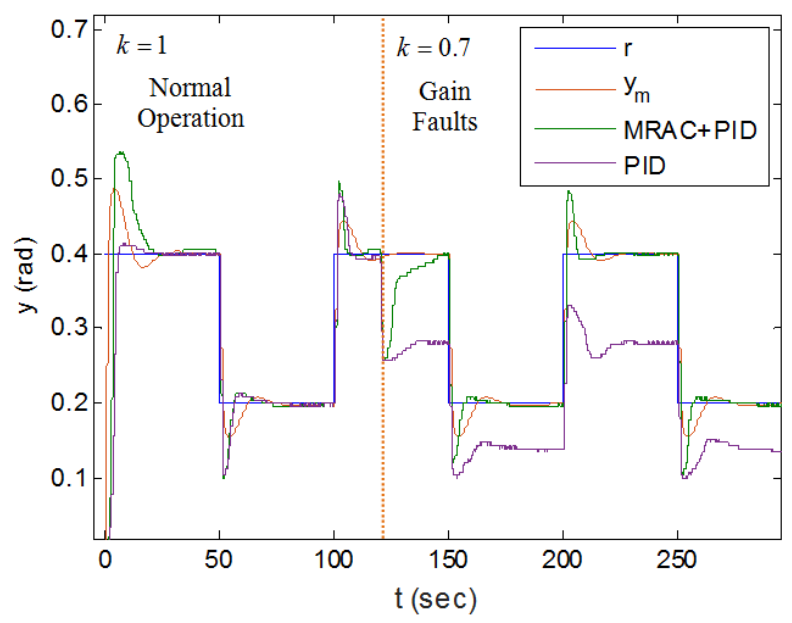

Fig. 9. Responses of multi-loop MRAC-PID system and conventional PID control system in the case of a gain fault of the closed loop PID control system at $120 \mathrm{sec}$.

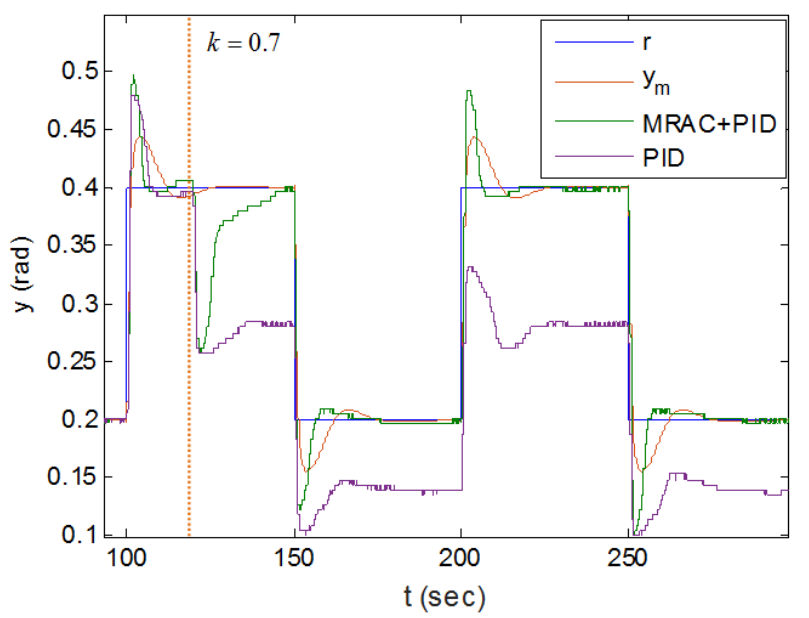

Fig. 10. A close view of Fig.9 to show responses of both control systems at the beginning of gain fault state at $120 \mathrm{sec}$.

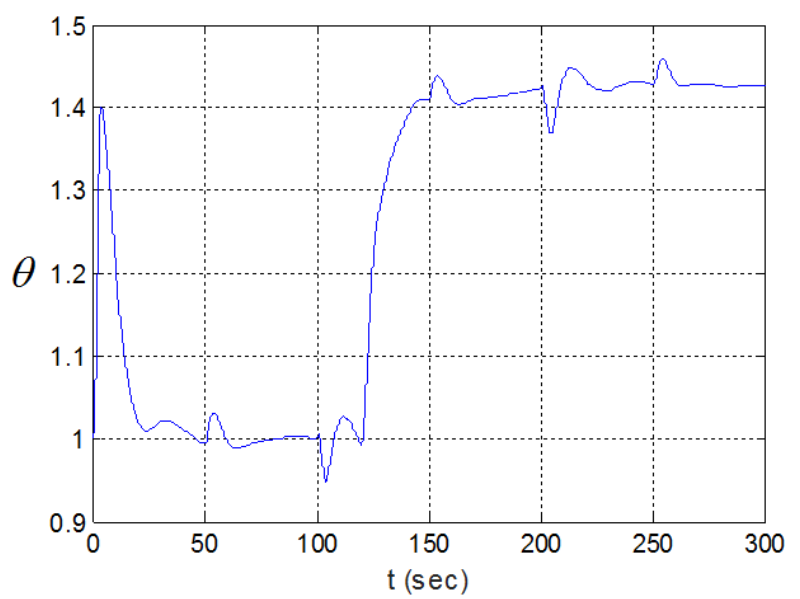

Fig. 11. Change of $\theta$ during experimental test of multi-loop MRAC-PID system

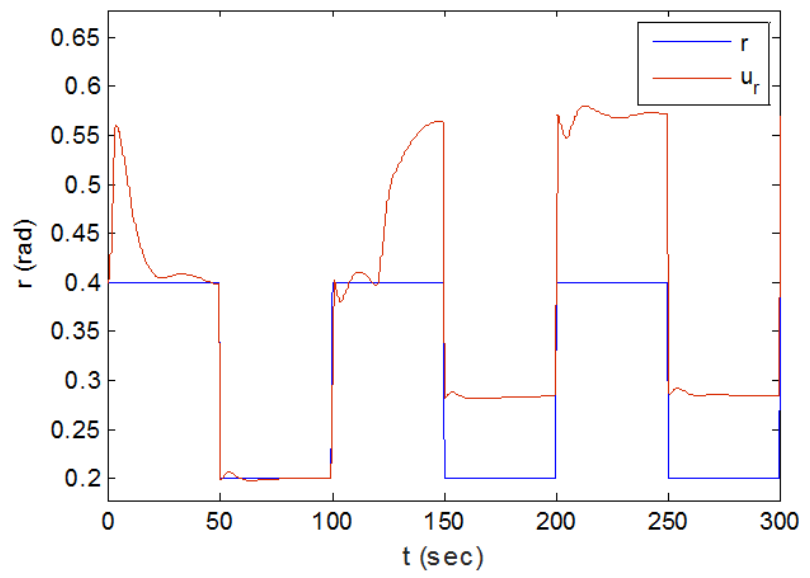

Fig. 12. $u_{r}$ during experimental test of multi-loop MRAC-PID system

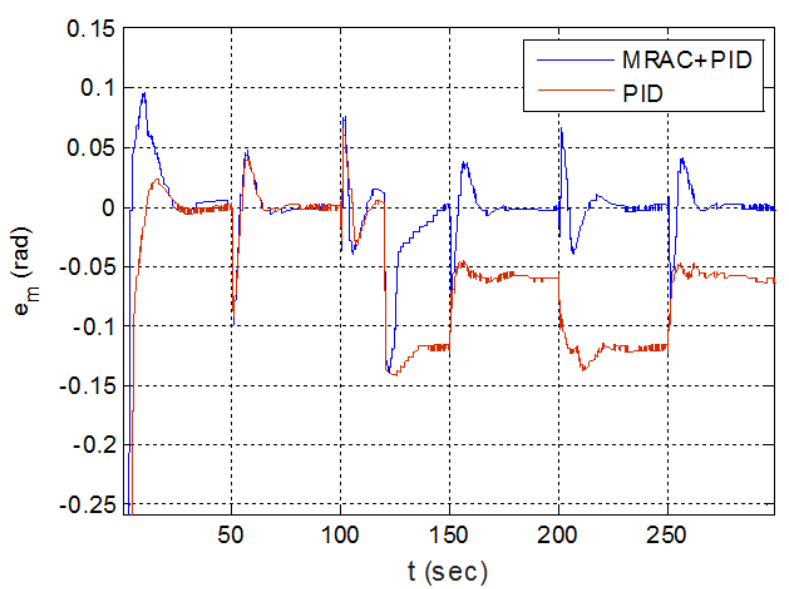

Fig. 13. Change of $e_{m}$ during experimental test of multi-loop MRAC-PID system and conventional PID control system. 


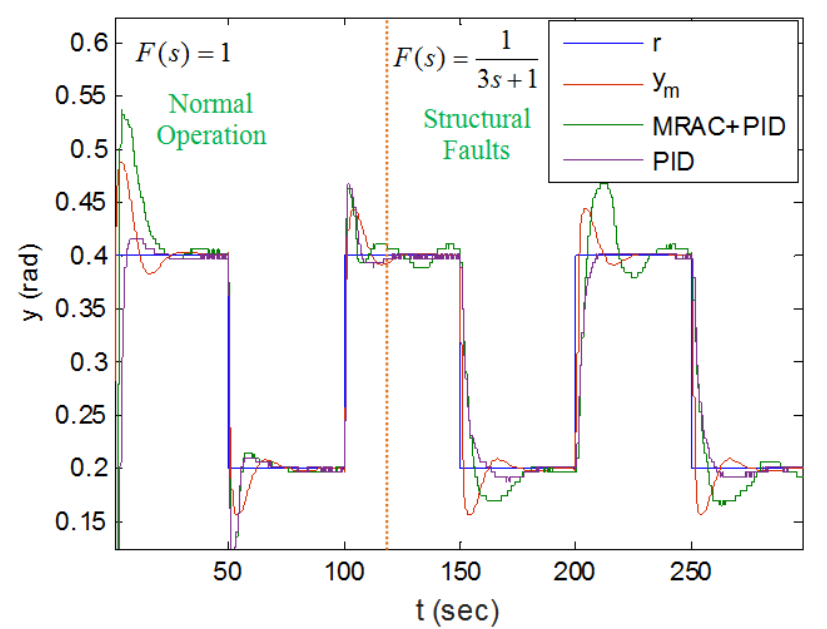

Fig. 14. Responses of multi-loop MRAC-PID system and conventional PID control system in the case of a structural fault of the closed loop PID control system at $120 \mathrm{sec}$

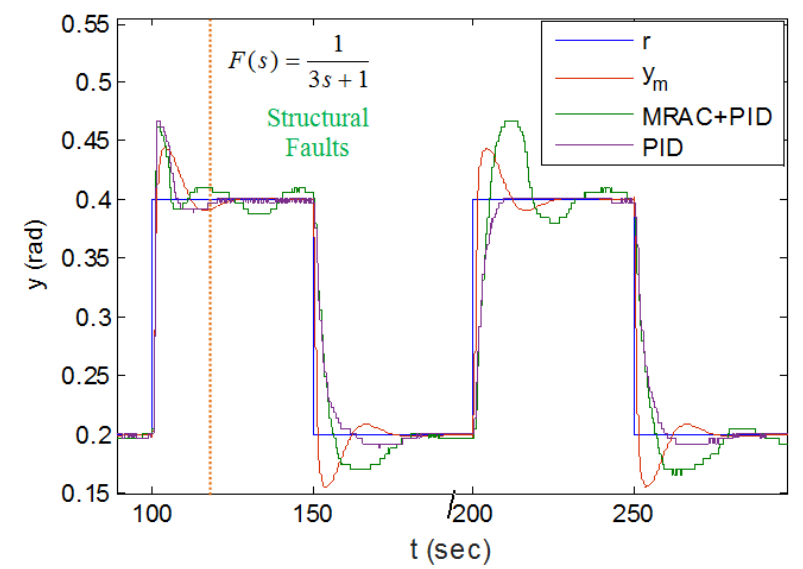

Fig. 15. A close view of Fig.14 to show responses of both control systems at the beginning of gain fault state at $120 \mathrm{sec}$.

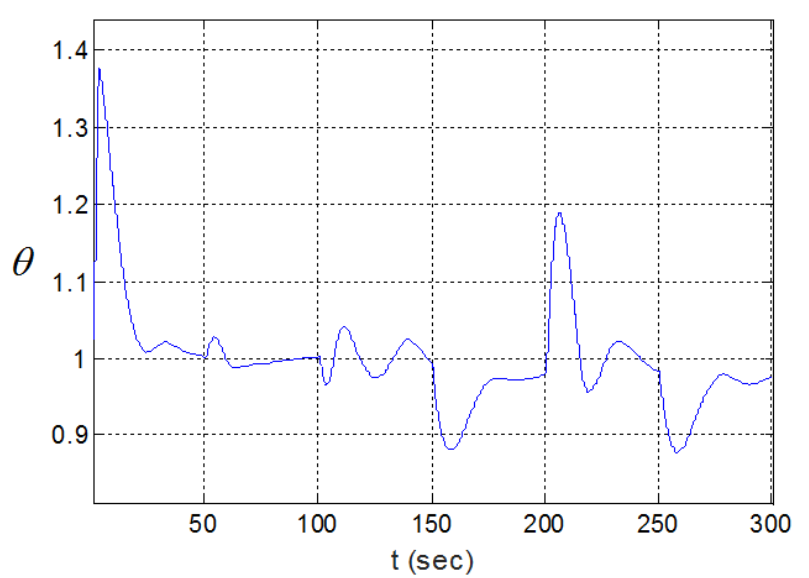

Fig. 16. Change of $\theta$ during experimental test of multi-loop MRAC-PID system

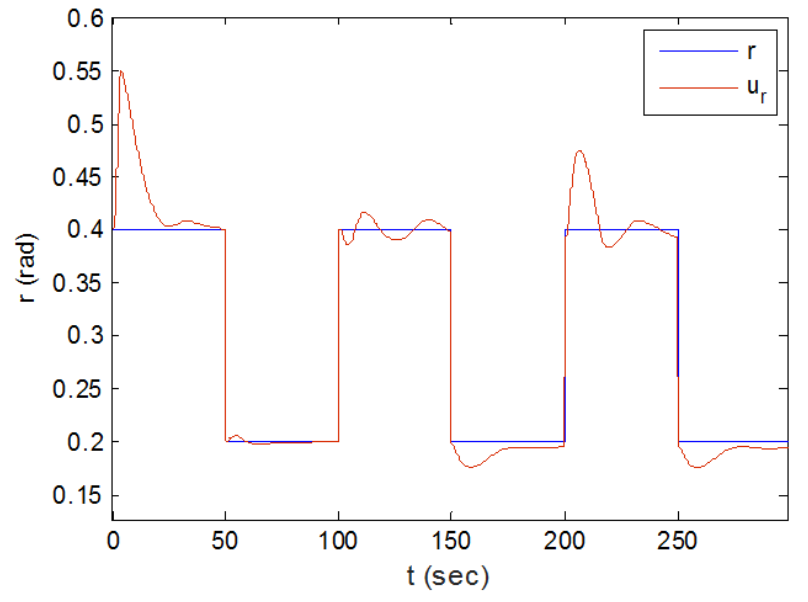

Fig. 17. Change of $u_{r}$ during experimental test of multi-loop MRAC-PID system

\section{CONCLUSIONS}

This study demonstrated experimental study for application of MRAC-PID structure to implement adaptive PID control of electrical rotors. Transformation of the existing and operational PID control systems to multi-loop MRAC-PID structure is quite straightforward task, and this multi-loop structure has potential of enhancement of robust control performance of existing PID control systems. Numerical and experimental study results clearly show that the proposed approach can increase adaptation and fault tolerance capability of conventional PID control systems without need for any modification on parameters of PID control systems. In real applications, the closed-loop PID control can compensate the developing faults until they develop a serious failure of control system. The MRAC-PID structure allows detection and diagnosis of fault status of closed loop control systems by observing the state of $\theta$ signals. This property may have significance for mission-critical control. Some remarks can be summarized:

* Simulation and experimental results show that the proposed multi-loop MRAC-PID system can be effective to compensate control performance deteriorations that are caused by gain type faults.

* Implementation of the method is very straightforward; it can be applied to the existing PID control systems only by performing closed loop model identification. No need for modification of closed loop PID control loop. This is an important asset for improvement of exiting control systems.

* Another practical advantage of this multi-loop MRAC-PID structure comes from performing reference input-shaping instead of dynamically updating PID controller coefficients for retuning the controller. This property is very desirable for mission-critical and remote control applications because online returning actions of controller coefficients can raise the risk of instantaneous instability or performance deteriorations. * Under harsh conditions, ageing or failing of system components can occur rather faster than normal conditions. 
Fault tolerance capability of control systems can contribute to robust performance of control systems by dealing with such performance deteriorations that are caused from instantaneous or slowly developing faults or defects of system components. The multi-loop MRAC-PID control can contribute to maintain control performance in industrial application.

\section{Acknowledgement}

This research is supported by TUBITAK [grand number 215E261].

\section{References}

[1] M. Blanke, M. Staroswiecki, N. E. Wu, "Concepts and methods in faulttolerant control," Proc. ACC, vol.4, pp. 2606-2620, June, 2001.

[2] A. L. Salih, M. Moghavvemi, H. A. Mohamed, K. S. Gaeid, "Flight PID controller design for a UAV quadrotor," Scientific Research and Essays, vol. no. 23, pp.3660-3667, 2010.

[3] N. Hengameh, H. Kharrati, "PID controller design for unmanned aerial vehicle using genetic algorithm," $23^{\text {rd }}$ International Symposium on Industrial Electronics, pp.213-217, June, 2014.

[4] K. J. Astrom, T. Hagglund, "The future of PID control," Control Engineering Practice, vol.9, no. 11, pp. 1163-1175, 2001.

[5] P. V. Osburn, H. P. Whitaker, A. Kezer, "Comparative studies of systems, "Institute of Aeronautical Science, pp. 61-39, 1961.

[6] I. D. Landau I.D, Adaptive Control the Model Reference Approach. New York: Marcel Dekker, 1979.

[7] K. J. Astrom, B. Wittenmark, Adaptive Control. Massachusetts: AddisonWesley, 1995.

[8] H. Butler, Model-Reference Adaptive Control-From Theory to Practice. New Jersey: Prentice-Hall, 1992.

[9] B. M. Vinagre, I. Petrás, I. Podlubny, Y. Q. Chen, "Using fractional order adjustment rules and fractional order reference models in model-reference adaptive control," Nonlinear Dynamics, vol. 29, no. 1-4, pp.269-279, 2001.

[10] E. Lavretsky, Adaptive control: Introduction, overview, and applications. Adaptive Control Workshop, NASA Marshall Space Center, USA, 2009.

[11] P. Jain, M.J. Nigam, "Design of a model reference adaptive controller using modified MIT rule for a second order system," Advance in Electronic and Electric Engineering, 3(4), 477-484, 2013.

[12] M.D. Bernardo, U. Montanaro, J.M. Olm S. Santini, "Model reference adaptive control of discrete time piecewise linear systems," International Journal of Robust and Nonlinear Control, vol. 23i no. 7, pp. 709-730, 2013.

[13] B. Jiang, Z. Gao, P. Shi, Y. Xu, "Adaptive fault-tolerant tracking control of near-space vehicle using Takagi-Sugeno fuzzy models," IEEE Transactions on Fuzzy Systems, vol. 18, no. 5, pp. 1000-1007, 2010.

[14] I. Sadeghzadeh, A. Mehta, Y. Zhang, C. Rabbath, "Fault-tolerant trajectory tracking control of a quadrotor helicopter using gain-scheduled PID and model reference adaptive control," Annual Conference of the Prognostics and Health Management Society, Montreal, Canada, pp.1-10, 2011.

[15] BB. Alagoz, A. Ates, C. Yeroglu, "Auto-tuning of PID controller according to fractional-order reference model approximation for DC rotor control," Mechatronics, vol. 23, no. 7, pp. 789-797, 2013.

[16] A. Znidi, K. Dehri, A. S. Nouri, "Discrete variable structure model reference adaptive control using only input-output measurements," Transactions of the Institute of Measurement and Control, vol.40, no. 3, pp. 861-872., 2018.

[17] B. Sinafar, A. R. Ghiasi, A. K. Fazli, "A new model reference adaptive control structure for uncertain switched systems with unmodeled input dynamics," Transactions of the Institute of Measurement and Control, vol. 37, no. 10, pp. 1171-1180, 2015.

[18] R. D. Tehrani, F. Shabaninia, "Two-level control of photovoltaic systems using global perturbation-based extremum seeking control and model reference adaptive control," Transactions of the Institute of Measurement and Control, vol. 40, no. 13, pp. 3709-3720.

[19] P. Sarhadi, A. R. Noei, A. Khosravi, "Model reference adaptive PID control with anti-windup compensator for an autonomous underwater vehicle," Robotics and Autonomous Systems, vol.83, pp. 87-93, 2016.

[20] W. Su, "A model reference-based adaptive PID controller for robot motion control of not explicitly known systems," International Journal of Intelligent Control and Systems, vol. 12, pp.237-244, 2007.
[21] Xiao S., Li Y. and Liu J.: A model reference adaptive PID control for electromagnetic actuated micro-positioning stage. 8th IEEE International Conference on Automation Science and Engineering, Seoul, Korea, pp. 97102., 2012.

[22] B. Singh, V. Kumar, "A real time application of model reference adaptive PID controller for magnetic levitation system," Power, Communication and Information Technology Conference, Bhubaneswar, India, pp.1-8., 2016.

[23] H. Noura, D. Theilliol, J. C. Ponsart, A. Chamseddine, Fault-tolerant Control Systems Design and Practical Applications. London: SpringerVerlag, 2009.

[24] G. J. J. Ducard, Fault-tolerant flight control and guidance systems practical methods for small unmanned aerial vehicles. London: SpringerVerlag, 2009.

[25] S. Ding, Y. Yuan, N. Xue, X. Liu, "Online fault-tolerant onboard aeroengine model tuning structure," International Journal of Aerospace Engineering, pp.1-16, 2016.

[26] W. M. Wonham, A control theory for discrete event system, advanced computing concepts and techniques in control engineering. Berlin: SpringerVerlag, pp.129-169., 1988.

[27] K. Wang, J. Chen, Z. Song, "Data-driven sensor fault diagnosis systems for linear feedback control loops," Journal of Process Control, vol.54, pp. 152-171, 2017.

[28] B. B. Alagoz, E. Petlenkov, C. Yeroglu, "Multi-loop model reference adaptive control of fractional-order PID control systems," 40th TSP, Barcelona, pp.702-705, July, 2017.

[29] A. Tepljakov, B. B. Alagoz, E. Gonzalez, E. Petlenkov, C. Yeroglu, "Model reference adaptive control scheme for retuning method-based fractional-order PID control with disturbance rejection applied to closed-loop control of a magnetic levitation system," Journal of Circuits Systems and Computers, vol.27, no.11, 2018.

[30] R. Rajesh, S. N. Deepa. "Design of Direct MRAC Augmented With 2 DoF PIDD Controller: An Application to Speed Control of a Servo Plant." Journal of King Saud University-Engineering Sciences, Available online 1 March 2019.

[31] N. Penkov, "A new Approach for Adaptive Tuning of PI Controllers," Application in Cascade Systems, Information Technologies and Control, vol. 6, no. 1 pp. 19-26, 2008.

[32] A. S. Alekseev, S. V. Zamyatin, V. A. Rudnicki, "Multi-loop control system design," Bulletin Of The Polish Academy Of Sciences, Technical Sciences, vol. 60: 627-630, 2012.

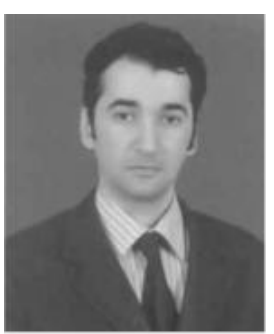

B.B. Alagoz was graduated from Istanbul Technical University department of Electronics and Communication Engineering in 1998. He has $\mathrm{PhD}$ degree from Inonu University, department of Electrical-Electronics Engineering in 2015. His research interests are intelligent systems, control, simulation and modeling.

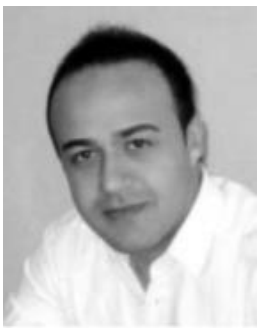

G. Kavuran received his B.Sc. degree in Electrical and Electronics Engineering from Firat University in 2008. He received $\mathrm{PhD}$ degree in Electrical and Electronics Engineering from Firat University in 2017. His research interests include robotics, fractional calculus, control theory and applications, modeling and simulation, signal processing.

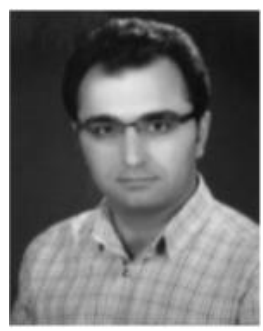

A. Ates received his B. Sc. Degree in Electrical and Electronics Engineering from Erciyes University in 2011. He received $\mathrm{PhD}$ degree in Computer Engineering from Inonu University in 2018 respectively. His research interests 
include optimization algorithm, analytical and numerical optimization method, fractional order control systems.

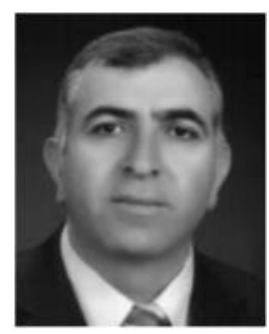

C. Yeroglu received his B.Sc. degree in Electrical and Electronics Engineering from Hacettepe University in 1990. He received his first Ph.D. degree in Computer Engineering from Trakya University in 2000 and his second Ph.D. degree in Electrical and Electronics Engineering from Inonu University in 2011. His research interests include fractional order control systems, robust control, nonlinear control, modeling and simulation, optimization algorithm.

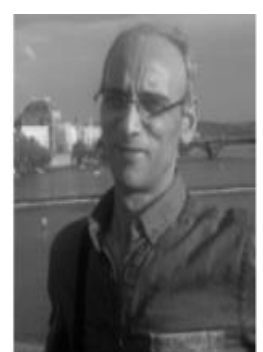

H. Alisoy was graduated from Moscow Technical University department of Electro-Physics Engineering 1982. He had his PhD degree from USSR Science Academy Physics Institute of P.N. Lebedyev and Doctor of Sciences degree (DSc) from International EcologyEnergy Academy. He works at Namik Kemal University, Department of Electronics and Communication Engineering. 